\title{
Local Dominance and Controlling Dominance Area of Solutions in Multi and Many Objectives EAs
}

\author{
Hiroyuki Sato* \\ Hernán E. Aguirre** \\ ${ }^{*}$ Faculty of Engineering, Shinshu University \\ Kiyoshi Tanaka* \\ **Fiber-Nanotech Young Researcher Empowerment Program, Shinshu University \\ 4-17-1 Wakasato, Nagano, 380-8553 JAPAN \\ \{sato@iplab., ahernan@,ktanaka@\}shinshu-u.ac.jp
}

\begin{abstract}
This work presents local dominance with alignment of principle search direction and control of dominance area of solutions to enhance selection of MOEAs, aiming to improve their performance on multi and many objectives combinatorial problems. We show that the methods used independently can substantially improve either diversity or convergence. Also, by including control of dominance area of solutions within the local dominance algorithm, we show that diversity and convergence can improve simultaneously while reducing the computational cost of the algorithm.
\end{abstract}

\section{Categories and Subject Descriptors}

I.2.8 [Artificial Intelligence]: Problem Solving, Control Methods, and Search-Heuristic methods; G.1.6 [Numerical Analysis]: Optimization

\section{General Terms}

Algorithms, Design, Performance

\section{Keywords}

Evolutionary multi and many objectives optimization, selection, local dominance, control of dominance area of solutions

\section{INTRODUCTION}

Multiobjective evolutionary algorithms (MOEAs) [1, 2] are being increasingly applied to solve multi-objective optimization problems. MOEAs aim to find a set of well distributed solutions that converge to the true Pareto front. MOEAs based on Pareto dominance perform effectively on problems with two and three objectives compared to conventional methods. However, satisfying simultaneously convergence and diversity requirements is difficult and often we have to sacrifice one for the other, especially on combinatorial problems. In addition, performance of current MOEAs drastically deteriorates for more than three objectives, rapidly approaching random search as the number of objectives increases. The reason is that the likelihood of solutions being Pareto non-dominated gets larger just by increasing the number of objectives, making conventional Pareto dominance selection useless because there is no way to rank and discriminate among solutions.

This work focuses on two methods designed to enhance selection in MOEAs aiming to increase their effectiveness

Copyright is held by the author/owner(s).

GECCO'08, July 12-16, 2008, Atlanta, Georgia, USA.

ACM 978-1-60558-131-6/08/07. on multi and many objectives combinatorial optimization problems. The first method is local dominance with alignment of principle search direction [3]. Local dominance induces the algorithm to search for solutions along the whole true Pareto front. However, convergence might slightly deteriorate in some regions. The other method is control of dominance area of solutions (CDAS) [4], which allows creating appropriate rankings of solutions to emphasize either diversity or convergence. CDAS is very effective in many objectives problems, where an appropriate ranking is specially required. By including CDAS within the local dominance algorithm, we show that convergence and diversity can improve simultaneously. In addition, local dominance with CDAS can be applied within very small neighborhoods, reducing significantly the computational cost of the algorithm. In this work we use $0 / 1$ multiobjective knapsack problems with up to 10 objectives and 500 items.

\section{LOCAL DOMINANCE MOEA}

The main steps of the local dominance MOEA [3] at each generation are as follows:

(i) Calculate Local Dominance $(L D)$ for each individual in the population $\mathcal{P}(t)$ and assign a local non-domination rank.

(ii) Assign a $\theta$-crowding factor to each individual.

(iii) Truncate the population $\mathcal{P}(t)$ to obtain the parent population $\mathcal{Q}(t)$ taking into account the local nondominance ranking and $\theta$-crowding factor.

(iv) Create the offspring population $\mathcal{R}(t)$ from $\mathcal{Q}(t)$ applying Local Recombination ( $L R$ ).

(v) Evaluate the offspring population $\mathcal{Q}(t)$.

(vi) Join the parent and offspring population to create the new population $\mathcal{P}(t+1)$ for the next generation.

\subsection{Local Dominance (LD)}

To calculate local dominance, first, the fitness vector of each individual in the population $\mathcal{P}(t)$ is transformed to polar coordinates, i.e. an individual $p$ is expressed by a norm $r_{p}$ and $m-1$ declination angles $\theta_{1, p}, \theta_{2, p}, \cdots, \theta_{m-1, p}$, where $m$ is the number of objectives. Second, the neighborhood for local dominance $S_{L D}$ of individual $p$ is temporally created as a local sub-population by choosing the $n_{L D}$ closest individuals to $p$ from the entire population $\mathcal{P}(t)$. Closeness $\delta_{p, x}$ between an individual $p$ and another individual $x$ is determined by their declination angles as follows

$$
\delta_{p, x}=\sum_{i=1}^{m-1}\left|\theta_{i, p}-\theta_{i, x}\right| .
$$




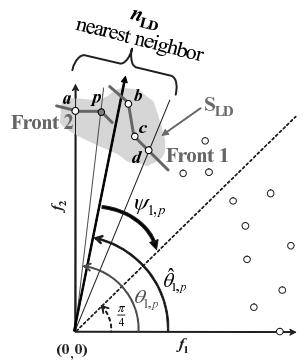

(a) Before rotation

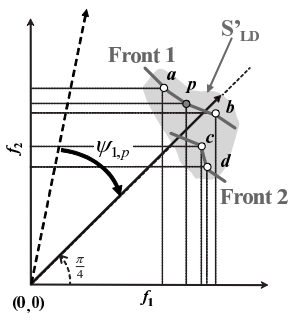

(b) After rotation

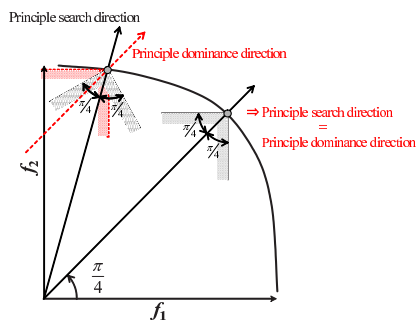

(c) Alignment

Figure 1: Neighborhood creation, its rotation, and the obtained fronts after non-dominance sorting in the calculation of Local Dominance (LD), (a) and (b). Alignment of principle dominance direction with principle search direction, (c).

Third, a principle search direction $\left\{\hat{\theta}_{1, p}, \hat{\theta}_{2, p}, \cdots, \hat{\theta}_{m-1, p}\right\}$ for the neighborhood $S_{L D}$ is established by calculating the angle difference between extreme individuals in $S_{L D}$. That is,

$$
\hat{\theta}_{i, p}=\frac{\left(\theta_{i, p}^{\max }-\theta_{i, p}^{\min }\right)}{2}+\theta_{i, p}^{\min }
$$

where $\theta_{i, p}^{\max }=\max \left\{\theta_{i, x_{1}}, \theta_{i, x_{2}}, \cdots, \theta_{i, x_{n_{L}}}\right\}, \theta_{i, p}^{\min }=\min$ $\left\{\theta_{i, x_{1}}, \theta_{i, x_{2}}, \cdots, \theta_{i, x_{n}}\right\}$, and $x_{j} \in S_{L D}$. Next, the principle search direction is rotated by $\left\{\hat{\theta}_{1, p}-\pi / 4, \hat{\theta}_{2, p}-\pi / 4, \cdots\right.$, $\left.\hat{\theta}_{m-1, p}-\pi / 4\right\}$, so that all its declination angles would be $\pi / 4$. Accordingly, all individuals in the local sub-population are rotated by the same rotation angles, so their declination angles would be around $\pi / 4$. Finally, non-domination sorting [1] is applied to the rotated neighborhood $S_{L D}^{\prime}$, and $p$ is assigned a rank equal to the non-dominated front it belongs to. Fig. 1 (a) and (b) illustrate for two objectives the neighborhood creation, its rotation, and the fronts obtained with non-domination sorting before and after rotation. Varying the number of elements in the neighborhood $n_{L D} \leq|\mathcal{P}(t)|$ we can control the degree of locality for dominance. In the extreme, $n_{L D}=|\mathcal{P}(t)|$, we have global dominance as in the case of conventional MOEAs.

\subsection{Motivation and Expected Effect}

The motivation to rotate the principle search direction of the neighborhood is to establish more precisely local nondomination relationships by aligning the direction where the individuals are leading (search direction) with the direction of their area of influence (dominance direction). The rotation angle is $\pi / 4$ because precisely at that angle the principle search direction of the sub-population is coincident with the principle dominance direction, as illustrated in Fig. 1 (c).

The rotation of the sub-population changes dominance relationships among solutions, increasing the chance of selecting promising diverse solutions. Let us illustrate this with an example. As shown in Fig. 1 (a), solutions $a$ and $p$ have the potential to disperse the search towards the direction of objective function $f_{2}$. However, if we calculate dominance with a conventional scheme, say NSGA-II [1], $a$ and $p$ would be assigned a lower rank and dismissed with high probability in the parent selection process since they appear globally dominated by $b$. On the contrary, if we take into account the principle search direction of $S_{L D}$ and properly rotate declination angles, as shown in Fig. 1 (b), $a$ and $p$ become non-dominated increasing the likelihood of finding better solutions in the direction of objective function $f_{2}$.

\subsection{Local Recombination (LR) and $\theta$-crowding}

In our method offspring are created one at the time. First, we specify a random principle search direction $\boldsymbol{\nu}=\left\{\nu_{1}, \nu_{2}\right.$, $\left.\cdots, \nu_{m-1}\right\}$, where $0 \leq \nu_{i} \leq \pi / 2$. Second, a neighborhood for local recombination $S_{L R}$ is temporally created as a local sub-population around $\boldsymbol{\nu}$ by choosing the $n_{L R}$ closest individuals to $\boldsymbol{\nu}$ from the parent population $\mathcal{Q}(t)$. Here $(1)$ is used with $\boldsymbol{\nu}$ instead of the declination angles of $p, \delta_{\boldsymbol{\nu}, x}$. Third, mating is performed within the neighborhood $S_{L R}$ and then recombination followed by mutation are carried out. Binary tournament selection is used for mating, but we enforce equal participation in the tournaments. Varying the number of elements in the neighborhood $n_{L R} \leq|\mathcal{Q}(t)|$ we can control the degree of locality for recombination. In the extreme, $n_{L R}=|\mathcal{Q}(t)|$, we have global recombination as in the case of conventional MOEAs.

$\theta$-crowding is used to calculate the crowding factor of each solution in our method [3]. $\theta$-crowding is inspired from the crowding distance procedure used by NSGA-II [1], but it is based on declination angles rather than on fitness values.

\section{CONTROL OF DOMINANCE AREA}

In general, the dominance area is uniquely determined with a fitness vector $\boldsymbol{f}(\boldsymbol{x})=\left(f_{1}(\boldsymbol{x}), f_{2}(\boldsymbol{x}), \cdots, f_{m}(\boldsymbol{x})\right)$ in the objective space when a solution $\boldsymbol{x}$ is given. To contract and expand the dominance area of solutions, we modify fitness value for each objective function by changing the user defined parameter $S_{i}$ in the following equation

$$
f_{i}^{\prime}(x)=\frac{r \cdot \sin \left(\omega_{i}+S_{i} \cdot \pi\right)}{\sin \left(S_{i} \cdot \pi\right)} \quad(i=1,2, \cdots, m)
$$

where $\varphi_{i}=S_{i} \cdot \pi$. This equation is derived from the Sine theorem. We illustrate the fitness modification in Fig. 2, where $r$ is the norm of $\boldsymbol{f}(\boldsymbol{x}), f_{i}(\boldsymbol{x})$ is the fitness value in the $i$-th objective, and $\omega_{i}$ is the declination angle between $\boldsymbol{f}(\boldsymbol{x})$ and $f_{i}(\boldsymbol{x})$. In this example, the $i$-th fitness value $f_{i}(\boldsymbol{x})$ is increased to $f_{i}^{\prime}(\boldsymbol{x})>f_{i}(\boldsymbol{x})$ by using $\varphi_{i}<\pi / 2\left(S_{i}<\right.$ $0.5)$. In case of $\varphi_{i}=\pi / 2\left(S_{i}=0.5\right), f_{i}(\boldsymbol{x})$ does not change and $f_{i}^{\prime}(\boldsymbol{x})=f_{i}(\boldsymbol{x})$. Thus, this case is equivalent to the conventional dominance. On the other hand, in case of $\varphi_{i}>$ $\pi / 2\left(S_{i}>0.5\right), f_{i}(\boldsymbol{x})$ is decreased so $f_{i}^{\prime}(\boldsymbol{x})<f_{i}(\boldsymbol{x})$.

Such fitness modification changes the dominance area of solutions. We show an example in Fig. 3, where three solutions $\boldsymbol{a}, \boldsymbol{b}$ and $\boldsymbol{c}$ are distributed in 2-dimensional objective space. In Fig. 3(a), $\boldsymbol{a}$ dominates $\boldsymbol{c}$, but $\boldsymbol{a}$ and $\boldsymbol{b}$, and $\boldsymbol{b}$ and $\boldsymbol{c}$ do not dominate each other. However, if we modify fitness values for each solution by using (3), the location of each solution moves in the objective space, and consequently the 


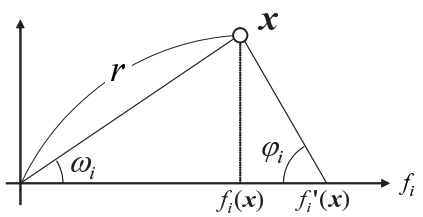

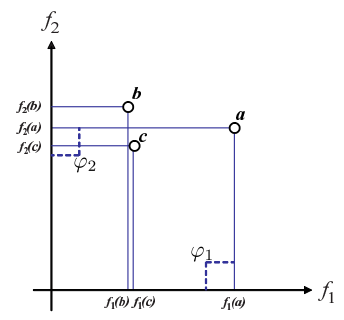

(a) $S_{1}=S_{2}=0.5$

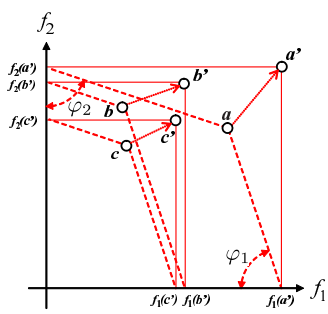

(b) $S_{1}=S_{2}<0.5$

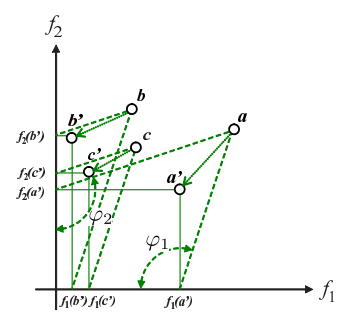

(c) $S_{1}=S_{2}>0.5$
Figure 2: Fitness modification

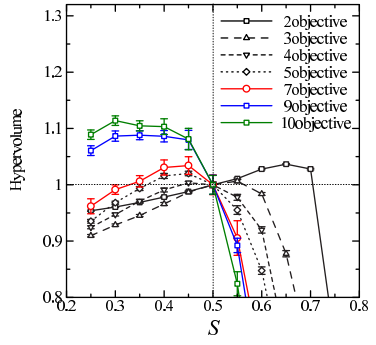

(a) NSGA-II with CDAS

Figure 3: Conventional dominance and examples of expanding and contracting the dominance area of solutions

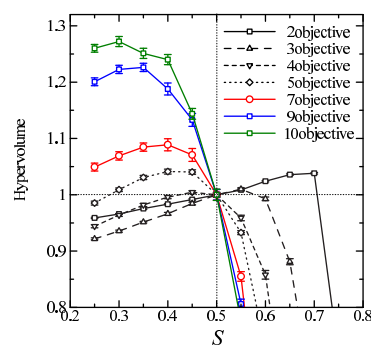

(b) SPEA2 with CDAS

Figure 4: Changes in hypervolume varying the parameter $S$ on problems with $m=\{2,3,4,5,7,9,10\}$, $n=500$ items, and $\phi=0.5$ feasibility ratio

dominance relationship among solutions changes. For example, if we use $S_{1}=S_{2}<0.5$ as shown in Fig. 3(b), the dominance area of solutions $\boldsymbol{a}^{\prime}, \boldsymbol{b}^{\prime}$ and $\boldsymbol{c}^{\prime}$ is expanded from the original one of $\boldsymbol{a}, \boldsymbol{b}$ and $\boldsymbol{c}$. This causes that $\boldsymbol{a}^{\prime}$ dominates $\boldsymbol{b}^{\prime}$ and $\boldsymbol{c}^{\prime}$, and $\boldsymbol{b}^{\prime}$ dominates $\boldsymbol{c}^{\prime}$. That is, expansion of dominance area by smaller $S_{i}(<0.5)$ works to produce a more fine grained ranking of solutions and would strengthen selection especially of solutions with higher projection on the $\pi / 4$ direction (middle regions of objective space). On the other hand, if we use $S_{1}=S_{2}>0.5$ as shown in Fig. 3(c), the dominance area of solutions $\boldsymbol{a}^{\prime}, \boldsymbol{b}^{\prime}$ and $\boldsymbol{c}^{\prime}$ is contracted from the original one of $\boldsymbol{a}, \boldsymbol{b}$ and $\boldsymbol{c}$. This causes that $\boldsymbol{a}^{\prime}, \boldsymbol{b}^{\prime}$ and $\boldsymbol{c}^{\prime}$ do not dominate each other. That is, contracting the area of dominance by larger $S_{i}(>0.5)$ works to produce a coarse ranking of solutions and would weaken selection by giving high rank to solutions located towards the extreme regions.

\section{RESULTS AND DISCUSSION 4.1 MOEA with CDAS}

In this paper we use multiobjective $0 / 1$ knapsack problems and evaluate the search performance of MOEAs with the hypervolume $(H V)$ and $\mathcal{C}$ metrics. The MOEAs are set with population size $|P|=200$, two-point crossover rate $p_{c}=1.0$, and bit-flipping mutation rate $p_{m}=1 / n$, where $n$ is the bit string length. We report the average performance on 30 runs, each of which spent $G=2,000$ generations. A constant strength $S=S_{i}(i=1,2, \cdots, m)$ for all objectives is used to control the dominance area of solutions.

First, we verify the effectiveness of CDAS on problems with up to 10 objectives. Fig. 4 (a) and (b) show the $H V$ achieved by NSGA-II and SPEA2, respectively, varying the value of the parameter $S$ in the range $[0.25,0.75]$ in intervals of 0.05 . In these figures, the $H V$ values are normalized so that the values achieved with conventional Pareto dominance $(S=0.5)$ are always 1.0 .

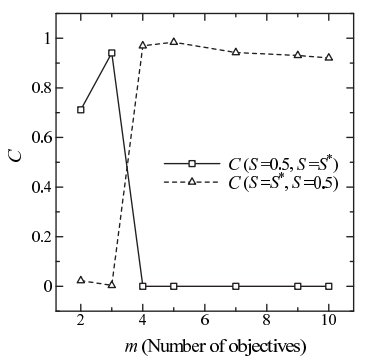

(a) NSGA-II

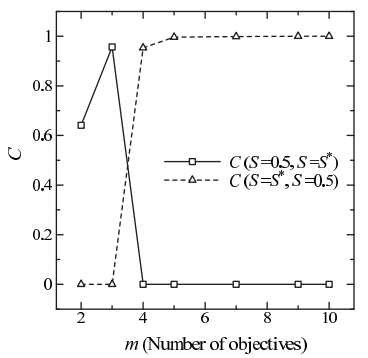

(b) SPEA2

Figure 5: $\mathcal{C}$ metric by conventional Pareto dominance $(S=0.5)$ and by CDAS set with optimum parameter that maximizes hypervolume $\left(S=S^{*}\right)$

From these figures important observations are as follows. First, for each number of objectives there is an optimum value $S^{*}$ that maximizes $H V$, which is higher (better) than the $H V$ achieved by conventional dominance at $S=0.5$. Second, maximum values of $H V$ are achieved for $m=\{2,3\}$ objectives by contracting the dominance area of solutions $(S>0.5)$ (weakening selection pressure), whereas for $m \geq 4$ the maximum $H V$ values are achieved by expanding the dominance area of the solutions $(S<0.5)$ (strengthening selection). As a general tendency, the optimum parameter $S^{*}$ that maximizes $H V$ gradually shifts towards smaller values as we increase the number of objectives. Third, the increase in $H V$ by CDAS is most significant for $m=\{9,10\}$ objectives. Fourth, SPEA2 shows a relative improvement larger than NSGA-II when the number of objectives increases.

To analyze with more detail the increase in $H V$ by CDAS, Fig. 5 (a) and (b) show the values of the $\mathcal{C}$ metric by conventional Pareto dominance $(S=0.5)$ and by CDAS set with the optimum parameter that maximizes $H V\left(S=S^{*}\right)$. Note that in $m=\{2,3\}$ objectives, most solutions obtained with controlled dominance $\left(S=S^{*}\right)$ are dominated by solutions obtained with conventional dominance $(S=0.5)$. Thus, for $m=\{2,3\}$ the increase of $H V$ by using $S^{*}>0.5$, which contracts dominance area of solutions making parent selection weaker, is due to a better diversity of solutions at the expense of convergence. On the other hand, for $m \geq 4$, solutions by controlled dominance $\left(S=S^{*}\right)$ dominate almost all solutions found by conventional dominance $(S=0.5)$. Thus, for $m \geq 4$ the increase of $H V$ by using $S^{*}<0.5$, which expands dominance area of solutions making parent selection stronger, is due to a better convergence. Note that a similar tendency is observed in both NSGA-II and SPEA2.

\subsection{Local Dominance MOEA with CDAS}

We examine the performance of the local dominance MOEA including CDAS after rotation of the principle search direc- 


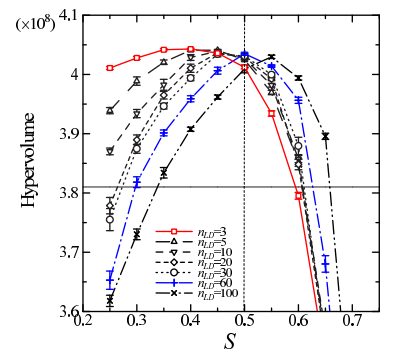

(a) KP500-2

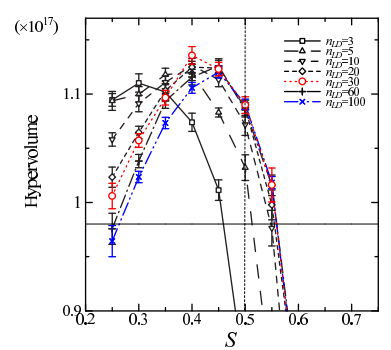

(c) KP500-4

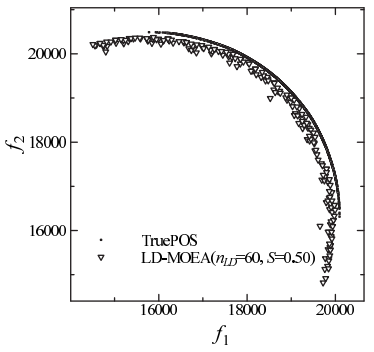

(e) $n_{L D}=60, S=0.50$

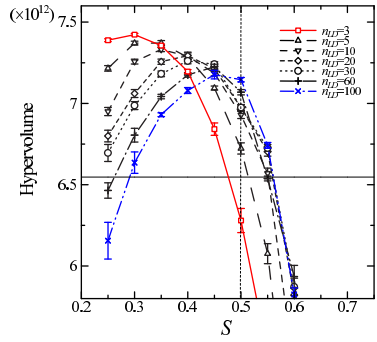

(b) KP500-3

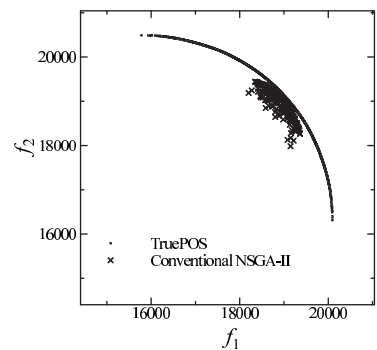

(d) NSGA-II

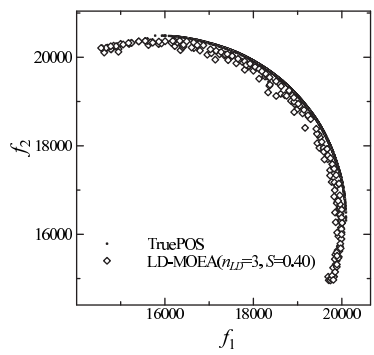

(f) $n_{L D}=3, S=0.40$
Figure 6: Results on Hypervolume (a)-(c). Final population obtained in a single run (d)-(f), KP5002

tion, before non-domination sorting of the local population. Here we use problems with $m=\{2,3,4\}$ objectives setting population sizes to $|P|=\{200,600,600\}$ and recombination neighborhood-size $n_{L R}=\{10,4,4\}$, respectively. Results on $H V$ are shown in Figure 6 (a)-(c) for dominance neighborhood-size $n_{L D}=\{3,5,10,20,30,60,100\}$. The horizontal lines in the figures show results by conventional NSGA-II.

First, looking at $S=0.5$ (on the dotted vertical line), we can see results by the algorithm that includes local dominance, but ranks solutions using conventional-Pareto dominance. Note that the local dominance MOEA achieves significantly better $H V$ than conventional NSGA-II even when very small neighborhoods are used. Second, comparing with the results at $S=0.5$, we can see that local dominance with expansion of dominance area of solutions $(S<0.5)$ can achieve higher values of $H V$. Note that this effect is more notorious for $m=\{3,4\}$ objectives than for $m=2$ objectives. Third, expansion of dominance area of solutions allows significant reductions on the size of the neighborhood, which reduces computation time of the local dominance MOEA. Note that the dominance neighborhood-sizes that maximize $H V$ are $n_{L D}^{H \max }=\{60,100,100\}$ by local conventional-Pareto dominance $(S=0.5)$, and $n_{L D}^{H \max }=$ $\{3,3,30\}$ by local dominance with expansion of dominance area $(S<0.5)$ for $m=\{2,3,4\}$ objectives, respectively.

Increases on $H V$ can be due to better diversity and/or

\begin{tabular}{|c|c|c|c|}
\hline \multicolumn{4}{|c|}{ Algorithms' parameters } \\
\hline \multirow[b]{2}{*}{ Algorithm } & \multicolumn{3}{|c|}{ Objectives } \\
\hline & $m=2$ & $m=3$ & $m=4$ \\
\hline A & $n_{L D}=60, S=0.5$ & $n_{L D}=100, S=0.5$ & $n_{L D}=100, S=0.5$ \\
\hline B & $n_{L D}=3, S=0.4$ & $n_{L D}=3, S=0.3$ & $n_{L D}=30, S=0.4$ \\
\hline \multicolumn{4}{|c|}{ Results on $C$ metric } \\
\hline \multirow[b]{2}{*}{$C$} & \multicolumn{3}{|c|}{ Objectives } \\
\hline & $m=2$ & $m=3$ & $m=4$ \\
\hline$C(A, B)$ & 0.172414 & 0.000000 & 0.006740 \\
\hline$C(B, A)$ & 0.689840 & 1.000000 & 0.818259 \\
\hline
\end{tabular}

Table 1: Results on $C$ metric by local dominance MOEA with and without expansion of dominance area of solutions

to better convergence. To analyze this better, Figure 6 (d)-(f) plot the final population of solutions obtained in a single run by three algorithms. From Figure 6 (d) note that NSGA-II's solutions converge close to the true Pareto front, but cover only a very narrow region of it. From Figure 6 (e) we can see that the local dominance algorithm $\left(S=0.5, n_{L D}^{H \max }=60\right)$ can find solutions covering the whole true Pareto front. Furthermore, as shown in Figure 6 (f), by including expansion of dominance area of solutions within the local dominance algorithm $\left(S=0.4, n_{L D}^{H \max }=3\right)$, the good diversity properties can be kept while converging closer to the true Pareto front. Moreover, Table 1 shows results on the $C$ metric obtained with dominance neighborhood sizes that maximize $H V n_{L D}^{H \max }$. From the table, looking at $C(B, A)$ values, we can see that most solutions obtained without expansion $(S=0.5)$ are dominated by solutions obtained with expansion of dominance area of solutions $(S<0.5)$, i.e. $70 \%, 100 \%$, and $82 \%$ on $m=\{2,3,4\}$ objectives, respectively. These results confirm that the gain on $H V$ is mostly due to a better convergence by including expansion of area of dominance of solution within the local dominance MOEA.

\section{CONCLUSIONS}

In this work we have presented local dominance with alignment of principle search direction and control of dominance area of solutions to enhance selection in MOEAs. We verified that the methods can substantially improve either diversity or convergence in problems with up to 10 objectives. Also, by including control of dominance area of solutions within the local dominance MOEA, we showed that diversity and convergence can be improved simultaneously while reducing computational time. As future works, we would like to study the combined method on continuous problems and apply it to real world applications.

\section{REFERENCES}

[1] K. Deb, Multi-Objective Optimization using Evolutionary Algorithms, John Wiley \& Sons, 2001.

[2] C. Coello, D. Van Veldhuizen, and G. Lamont, Evolutionary Algorithms for Solving Multi-Objective Problems, Boston, Kluwer Academic Publishers, 2002.

[3] H. Sato, H. Aguirre, and K. Tanaka, "Local Dominance and Local Recombination in MOEAs on 0/1 Multiple Knapsack Problems", European Journal of Operational Research, Vol. 181, No. 3, pp. 1708-1723, 2007.

[4] H. Sato, H. Aguirre, and K. Tanaka, "Controlling Dominance Area of Solutions and its Impact on the Performance of MOEAs", Proc. Fourth Intl. Conf. on Evolutionary Multi-Criterion Optimization, Springer, LNCS, Vol. 4403, pp. 5-20, 2007. 